\title{
Immunofluorescence microscopy for the rapid diagnosis of melioidosis
}

A L Walsh, M D Smith, V Wuthiekanun, Y Suputtamongkol, V Desakorn, W Chaowagul, N J White

\begin{abstract}
A direct immunofluorescent antibody test (DIF) was developed for the rapid diagnosis of melioidosis, a potentially fatal infection caused by Pseudomonas pseudomallei. In a clinical evaluation of 369 sputum, pus, or urine specimens from 272 patients with suspected melioidosis, the DIF had a sensitivity of $73 \%$ and a specificity of $99 \%$ compared with culture. Using this DIF, a confident diagnosis of melioidosis can now be made within two hours of admission to hospital, compared with the delay of two to four days required for culture results. Consequent early institution of specific antimicrobial therapy may help to save lives.
\end{abstract}

$(\mathcal{O}$ Clin Pathol 1994;47:377-379)

Pseudomonas pseudomallei is an important human and animal pathogen in Southeast Asia and northern Australia. ${ }^{12}$ In Thailand, most human melioidosis is seen in the large rice farming community in the northeastern region of the country, where it accounts for $20 \%$ of community acquired septicaemias. ${ }^{3}$ The spectrum of disease ranges from life threatening severe sepsis to mild localised infections. The clinical manifestations of melioidosis are protean, making a diagnosis difficult on clinical grounds alone. The mortality of severe disease remains high, despite recent improvements in treatment. ${ }^{45}$ Ps pseudomallei is intrinsically resistant to the commonly used empirical treatment for severe sepsis in Thailand, a penicillin plus gentamicin, and culture techniques require at least 48 hours to identify $P$ pseudomallei in clinical specimens. ${ }^{6}$ Delays in diagnosis could prove fatal, and therefore the use of rapid diagnostic methods is important. A direct immunofluorescent antibody test (DIF) for the rapid detection of Ps pseudomallei in clinical samples has been developed.

\section{Methods}

\section{CONJUGATE PREPARATION}

Rabbits were immunised with a heat killed suspension $\left(10^{9}\right.$ colony forming units $\left./ \mathrm{ml}\right)$ of Ps pseudomallei (strain 204, a clinical isolate from Ubon Ratchatani, Thailand) given intravenously in increasing doses every three to four days. Serum was collected three days after the fifth dose and fractionated (by protein A-sepharose CL-4B chromatography) to obtain purified polyclonal anti-Ps pseudomallei immunoglobulin G (IgG). Using the method of Samuel et $a l_{,}{ }^{7}$ the antibody was then conjugated to fluorescein isothiocyanate (FITC). In summary, the IgG was diluted in carbonate buffer at $\mathrm{pH} 9 \cdot 2$, FITC $(5 \mathrm{mg} / \mathrm{ml})$ was added in a 20:1 molar ratio and the mixture was left in the dark at room temperature for one hour. Unbound FITC was removed by filtration through Sephadex G25. Conjugate fractions were pooled and stored at $4{ }^{\circ} \mathrm{C}$ in phosphate buffered saline (PBS) containing $33 \%$ glycerol and $0.02 \%$ thiomersal.

\section{DIF METHOD}

Air dried smears were fixed in methanol for 30 seconds. The slides were flooded with blocking buffer (PBS with 3\% bovine serum albumin and 3\% normal rabbit serum), incubated at room temperature for 30 minutes and rinsed with PBS. They were then flooded with a 1:400 dilution (in blocking buffer) of the conjugate, incubated at room temperature for 1 hour in the dark, and finally washed in PBS for 15 minutes before drying on a hotplate. After mounting in buffered glycerol (pH 9), the slides were examined by incident light fluorescence, using a $\times 100$ oil immersion lens. A positive result was observed when the periphery of the bacilli showed strong apple-green fluorescence. A known positive control slide was included in each batch of tests.

\section{LABORATORY EVALUATION}

Fifty-one isolates of $P S$ pseudomallei (from patients in northeastern Thailand), 22 strains of other pseudomonas species and 22 different Enterobacteriaceae were tested. All strains were identified by API 20NE or API 20E (BioMerieux UK Ltd, Basingstoke, UK), and ancillary tests as described previously. Suspensions of organisms were made in PBS, to a density of $10^{5} / \mathrm{ml}$. Aliquots of $5 \mu \mathrm{l}$ were then spotted onto teflon coated multiwell slides and air dried.

\section{CLINICAL EVALUATION}

Samples of sputum, pus, wound swabs, and urine were obtained from 272 patients with suspected melioidosis in Sappasitprasong Hospital, Ubon Ratchatani, Thailand. ${ }^{3}$ Two thin smears were made from each sample, one for Gram staining and the other for DIF. All specimens were inoculated onto selective and non-selective agar, and into a selective broth as described previously; ${ }^{6}$ pus and other

\author{
Correspondence to: \\ Ms A L Walsh \\ Accepted for publication \\ 14 October 1993 \\ Oxford OX3 9DU \\ A L Walsh \\ M D Smith
}


body fluids were also put into tryptic soy broth. All solid media were incubated for four days to allow for typical colonial morphology to develop. Enrichment broths were subcultured after 48 hours' incubation at $42^{\circ} \mathrm{C}$. Suspect colonies of Ps pseudomallei were identified by standard methods ${ }^{8}$ and latex agglutination. ${ }^{9}$

\section{Results}

In the initial evaluation using cultures of fully identified organisms, the DIF was $100 \%$ specific for Ps pseudomallei. All 51 strains of Ps pseudomallei exhibited positive fluorescence, while none of the other Pseudomonas spp. (including those strains of Ps cepacia which agglutinate non-specifically in the $P S$ pseudomallei latex test) $)^{9}$ or Enterobacteriaceae gave a positive result (table 1).

In the clinical study, Ps pseudomallei was isolated from 150 of 369 clinical specimens. One hundred and twelve were positive by DIF (table 2); two of these were false positives and 110 were true positives, giving an overall sensitivity of $73 \%$ compared with culture, with a specificity of $99 \%$. The false positives both occurred in sputum specimens: one subsequently grew Ps aeruginosa and the other Ps cepacia. Broth cultures of each organism were retested by DIF, but were consistently negative. Ps pseudomallei could not be isolated from any specimen from either patient. The positive and negative predictive values of DIF were $98 \%$ and $85 \%$, respectively. Gram negative rods were seen in $203(55 \%)$ specimens: 105 were negative by DIF and culture, two gave false positive fluorescence, and four specimens were DIF negative but subsequently grew Ps pseudomallei. Of these four, three specimens had a heavy growth of coliforms, while the fourth was cultured on selective media alone, so the presence of other organisms could not be determined. Of

Table 1 Immunofluorescence results for positive and negative control organisms

\begin{tabular}{lll}
\hline Organism & Number tested & Number positive \\
\hline Ps pseudomallei & 51 & 51 \\
Pseudomonas species & & \\
$\quad$ Ps cepacia & 8 & 0 \\
Ps aeruginosa & 3 & 0 \\
Ps stutzeri & 3 & 0 \\
$\quad$ Others & 8 & 0 \\
Enterobacteriaceae & & \\
$\quad$ Rebsiella spp. & 6 & 0 \\
Escherichia spp. & 3 & 0 \\
Enterobacter spp. & 3 & 0 \\
Citrobacter spp. & 3 & 0 \\
$\quad$ Proteus spp. & 2 & 0 \\
Others & 5 & 0 \\
\hline
\end{tabular}

Table 2 Immunofluorescence results for 369 clinical specimens

\begin{tabular}{lcccl}
\hline $\begin{array}{l}\text { Specimen type } \\
\text { (no. tested) }\end{array}$ & GNR seen & $\begin{array}{c}\text { DIF } \\
\text { positive }\end{array}$ & $\begin{array}{l}\text { Culture } \\
\text { positive }\end{array}$ & $\begin{array}{l}\text { Sensitivity of } \\
\text { DIF (\%) }\end{array}$ \\
\hline Sputum (155) & 117 & $52^{\star}$ & 62 & 81 \\
Wound swabs (68) & 20 & 21 & 38 & 55 \\
Pus or fluid (90) & 35 & 31 & 41 & 76 \\
Urine (56) & 31 & 8 & 9 & 89 \\
Overall (369) & 203 & 112 & 150 & 73 \\
\hline
\end{tabular}

GNR $=$ Gram negative rod
* Includes two false positives the $92 \mathrm{DIF}$ and culture positive specimens, only 41 (45\%) contained Gram negative rods which were described as "typical" or bipolar. Overall, Gram stain alone had a diagnostic sensitivity of $62 \%$ and a specificity of only $50 \%$. Gram negative rods were not seen in 18 of $110(16 \%)$ specimens truly positive by DIF. There were $40(27 \%)$ false negative DIF results. Of these, 16 specimens gave only light growth of Ps pseudomallei ( $<20$ colonies) on direct plating, 10 grew $<5$ colonies, and the remaining 14 were cultured only from selective enrichment broth.

\section{Discussion}

The majority of patients with melioidosis are not diagnosed until a positive culture result is obtained, even in endemic areas where the index of suspicion is high. Inappropriate treatment due to delayed diagnosis contributes to the high mortality of this disease. ${ }^{3}$ In films of pus or sputum, melioidosis is often diagnosed if bipolar staining ("safety-pin") Gram negative rods are seen, ${ }^{1}$ but this finding is not specific. In this study only $41 / 150$ (27\%) of specimens containing Ps pseudomallei had "typical" Gram negative rods on direct microscopy, and similar "safety-pin" bacilli were also seen in $9 \%$ of smears from specimens which grew other Gram negative organisms.

Culture remains the gold standard for the definitive diagnosis of melioidosis. Isolation of Ps pseudomallei from blood or purulent exudates is not difficult, but problems may be encountered with specimens likely to have a heavy contaminating flora, such as sputum and throat swabs. The relatively slow development of typical colonies, even on non-selective media, makes recognition of Ps pseudomallei amongst mixed growth difficult. ${ }^{5}$ If growth occurs only after enrichment, then the delay to positive culture will be prolonged from two to four days.

The DIF method was originally developed for use on environmental specimens and exudates from experimentally infected animals. ${ }^{10}$ Recently a DIF method was evaluated in a small number of cases of suspected human melioidosis (Naigowit P, et al. Abstract presented at conference on medical laboratory technology, 1991). Sensitivity was $83 \%$ but positive fluorescence occurred in 6/44 samples which did not grow Ps pseudomallei (although selective enrichment broths may not have been used); the specificity was therefore only $88 \%$. In the present study, specificity was $99 \%$ and sensitivity was $73 \%$. The negative predictive value for a specimen in which Gram negative rods were seen but the DIF was negative was $96 \%$. Cultures from the false DIF negatives indicated that $60 \%$ contained a very scanty number of organisms, $35 \%$ growing from enrichment broth only. It is estimated that $10^{4}-10^{5}$ organisms $/ \mathrm{ml}$ are required for detection by light or fluorescent microscopy. The two apparently false positives are more difficult to explain. False positive organisms were not encountered during 
the laboratory evaluation, but it should be noted that neither Pseudomonas mallei nor Legionella pneumophila, both of which are known to cause serological cross-reactions with Ps pseudomallei, were available for testing. It is possible that small numbers of $P_{s}$ pseudomallei may have been overgrown by the other Gram negative rods which were present in the specimens. One patient had received prior treatment with chloramphenicol, and it is also possible that Ps pseudomallei was present but failed to grow in the presence of the antibiotic.

It is now possible to have a specific result within two hours in three out of four patients with melioidosis, if appropriate specimens are available (in this study, about $85 \%$ of patients were found to have specimens suitable for DIF on admission). For patients with no focal lesions, detection of urinary antigen may provide a rapid diagnosis. Reagent costs for the DIF are low: approximately $£ 50$ for $1 \mathrm{ml}$ of conjugate (400 tests) and $£ 3$ for $100 \mathrm{ml}$ of blocking buffer. Unfortunately, the technique requires an expensive fluorescence microscope. However, the recent development of a simple filter which modifies a standard light microscope for fluorescent work ${ }^{11}$ may allow peripheral laboratories in endemic areas to use this methodology. This method may also be adaptable to non-fluorescent visualisation, i.e. using horseradish peroxidase labelled antibodies and an appropriate chromogenic substrate. These strategies are currently being investigated.
The authors wish to thank Mr Sayan Langla for technical assistance; Dr T L Pitt and Khun Pimjai Naigowit for expert advice and encouragement; and the Director, medical, laboratory and nursing staff of Sappasitprasong Hospital for their continuing support. This study was part of the WellcomeMahidol University, Oxford Tropical Medicine Research Programme, funded by the Wellcome Trust of Great Britain.

1 Leelarasamee A, Bovornkitti S. Melioidosis: review and update. Rev Infect Dis 1989;11:413-25.

2 Dance DAB. Melioidosis. Rev Med Microbiol 1990;1: 143-50.

3 Chaowagul W, White NJ, Dance DAB, Wattanagoon Y, Naigowit P, Davis TM, Looareesuwan S, Pitakwatchara N. Melioidosis: a major cause of community-acquired septicemia in northeastern Thailand. $\mathcal{F}$ Infect Dis 1989; 159:890-9.

4 White NJ, Dance DAB, Chaowagul W, Wattanagoon Y, Wuthiekanun V, Pitakwatchara N. Halving of mortality Wuthiekanun V, Pitakwatchara N. Halving of mortality
of severe melioidosis by ceftazidime. Lancet 1989;2: 697-701.

5 Sookpranee M, Boonma P, Susaengrat W, Bhuripanyo K, Punyagupta S. Multicentre prospective randomized trial comparing ceftazidime plus cotrimoxazole with chloramphenicol plus doxycycline and cotrimoxazole for amphenicol plus doxycycline and cotrimoxazole for Chemother 1992;36:158-62.

6 Wuthiekanun V, Dance DAB, Wattanagoon Y, Supputtamongkol Y, Chaowagul W, White NJ. The use of selective media for the isolation of Pseudomonas pseudomallei in clinical practice. $\mathcal{f}$ Med Microbiol 1990; pseudomall

7 Samuel D, Patt RJ, Abuknesha RA. A sensitive method of detecting proteins on dot and western blots using monoclonal antibodies to FITC. $\mathcal{F}$ Immunol Methods 1988;107:217-24.

8 Dance DAB, Wuthiekanun V, Naigowit P, White NJ. Identification of Pseudomonas pseudomallei in clinical practice: use of simple screening tests and API $20 \mathrm{NE}$. f Clin Pathol 1989;42:645-8.

9 Smith MD, Wuthiekanun V, Walsh AL, Pitt TL. Latex agglutination test for identification of Pseudomonas pseudomallei. $千$ Clin Pathol 1993;46:374-5.

10 Thomason BM, Moody MD, Goldman M. Staining bacterial smears with fluorescent antibody. II. Rapid detection of varying numbers of Malleomyces pseudomallei in contaminated materials and infected animals f Bacteriol 1956;72:362-7.

11 Kawamoto F. Rapid diagnosis of malaria by fluorescence microscopy with a light microscope and interference filter. Lancet 1991;337:200-2. 